\title{
Quantum criticality and nodal superconductivity in the FeAs-based superconductor $\mathrm{KFe}_{2} \mathrm{As}_{2}$
}

\author{
J. K. Dong, ${ }^{1}$ S. Y. Zhou, ${ }^{1}$ T. Y. Guan, ${ }^{1}$ H. Zhang, ${ }^{1}$ Y. F. Dai, ${ }^{1}$ X. Qiu, ${ }^{1}$ X. F. Wang,${ }^{2}$ Y. He, ${ }^{2}$ X. H. Chen, ${ }^{2}$ S. Y. Li $^{1, *}$ \\ ${ }^{1}$ Department of Physics, Surface Physics Laboratory (National Key Laboratory), \\ and Laboratory of Advanced Materials, Fudan University, Shanghai 200433, China \\ ${ }^{2}$ Hefei National Laboratory for Physical Science at Microscale and Department of Physics, \\ University of Science and Technology of China, Hefei, Anhui 230026, China
}

(Dated: October 29, 2018)

\begin{abstract}
The in-plane resistivity $\rho$ and thermal conductivity $\kappa$ of FeAs-based superconductor $\mathrm{KFe}_{2} \mathrm{As}_{2}$ single crystal were measured down to $50 \mathrm{mK}$. We observe non-Fermi-liquid behavior $\rho(T) \sim T^{1.5}$ at $H_{c_{2}}=5 \mathrm{~T}$, and the development of a Fermi liquid state with $\rho(T) \sim T^{2}$ when further increasing field. This suggests a field-induced quantum critical point, occurring at the superconducting upper critical field $H_{c_{2}}$. In zero field there is a large residual linear term $\kappa_{0} / T$, and the field dependence of $\kappa_{0} / T$ mimics that in $d$-wave cuprate superconductors. This indicates that the superconducting gaps in $\mathrm{KFe}_{2} \mathrm{As}_{2}$ have nodes, likely $d$-wave symmetry. Such a nodal superconductivity is attributed to the antiferromagnetic spin fluctuations near the quantum critical point.
\end{abstract}

PACS numbers: 74.70.Xa, 74.25.fc, 74.40.Kb, 74.20.Rp

When superconductivity emerges with the suppression of magnetism, for example in heavy-fermion and hightemperature cuprate superconductors, the spin fluctuations associated with a magnetic quantum critical point is usually considered as the pairing glue. This is also the case for the recently discovered FeAs-based hightemperature superconductors [1-4]. The parent compounds of the FeAs-based superconductors, for example $\mathrm{LaFeAsO}$ and $\mathrm{BaFe}_{2} \mathrm{As}_{2}$, are not superconducting and manifest antiferromagnetic (AF) order [5, 6]. With electron or hole doping, the AF order is suppressed and superconductivity emerges [1-4].

Spin fluctuations usually result in superconducting gaps with nodes, but it can also give nodeless superconducting gaps through interband interaction, termed

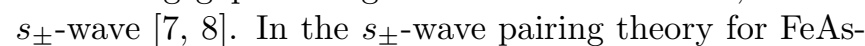
based superconductors 7], the interband interaction happens between the hole pockets at $\Gamma$ point and the electron pockets at $M$ point, via the antiferromagnetic spin fluctuations (AFSF) with wave vector $Q_{A F}=(\pi, \pi)$. This gives full superconducting gaps on both electron and hole pockets, but with the opposite signs of the order parameters between the two 7]. While there are accumulating experimental and theoretical works in favor of nodeless gaps, conclusive phase-sensitive experiments are needed to confirm the $s_{ \pm}$-wave pairing in FeAs-based superconductors [9]. Moreover, in LaFePO and $\mathrm{BaFe}_{2}\left(\mathrm{As}_{1-x} \mathrm{P}_{x}\right)_{2}$, two compounds containing phosphorus, there are evidences for nodal superconductivity $10-13]$. Therefore, the pairing symmetry and superconducting mechanism in iron pnictides are still far from consensus.

Recently, the ARPES experiments on the extremely hole-doped $\mathrm{KFe}_{2} \mathrm{As}_{2}\left(T_{c}=3 \mathrm{~K}\right)$ showed that the electron pockets at $\mathrm{M}$ point completely disappear due to the hole doping [14]. This result immediately raises a very important question: what is the superconducting state in heavily overdoped FeAs-based superconductors where the interband interaction is suppressed? While ARPES experiment was unable to study the superconducting gap structure in $\mathrm{KFe}_{2} \mathrm{As}_{2}$ due to its low $T_{c}$, the low-temperature thermal conductivity technique is particularly useful for studying exotic superconductors with low $T_{c}$ 15].

In this Letter, we report the demonstration of a clear field-induced antiferromagnetic quantum critical point and nodal superconductivity in $\mathrm{KFe}_{2} \mathrm{As}_{2}$ by resistivity and thermal conductivity measurements. Our findings not only confirm the spin-fluctuation-mediated pairing mechanism, but also clarify the pairing symmetry when interband interaction is suppressed in heavily overdoped regime, thus complete our understanding of the superconducting state in the FeAs-based superconductors.

Single crystals of $\mathrm{KFe}_{2} \mathrm{As}_{2}$ were grown by self-flux method [16]. The dc magnetic susceptibility was measured by a SQUID (Quantum Design). The sample was cleaved to a rectangular shape of dimensions $1.5 \times 1.0$ $\mathrm{mm}^{2}$ in the $a b$-plane, with $40 \mu \mathrm{m}$ thickness along the $c$-axis. Contacts were made directly on the sample surfaces with silver paint, which were used for both resistivity and thermal conductivity measurements. To avoid degradation, the sample was exposure in air less than 2 hours. The contacts are metallic with typical resistance $100 \mathrm{~m} \Omega$ at $1.5 \mathrm{~K}$. In-plane thermal conductivity was measured in a dilution refrigerator, using a standard four-wire steady-state method with two $\mathrm{RuO}_{2}$ chip thermometers, calibrated in situ against a reference $\mathrm{RuO}_{2}$ thermometer. Magnetic fields were applied along the $c$ axis and perpendicular to the heat current. To ensure a homogeneous field distribution in the sample, all fields were applied at temperature above $T_{c}$.

Fig. 1 shows the in-plane resistivity of our $\mathrm{KFe}_{2} \mathrm{As}_{2}$ single crystal. The residual resistivity ratio (RRR) $\rho(297$ 


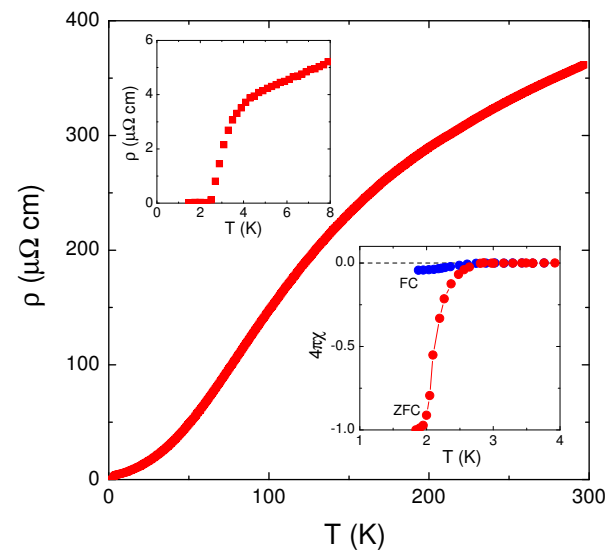

FIG. 1: (Color online). In-plane resistivity of $\mathrm{KFe}_{2} \mathrm{As}_{2}$ single crystal. The residual resistivity ratio is $\rho(297 \mathrm{~K}) / \rho(5 \mathrm{~K})$ $=86$. Upper inset: the resistive transition at low temperature. Lower inset: the dc magnetic susceptibility at $H=10$ Oe, with both zero field cooled (ZFC) and field cooled (FC) measuring conditions.

$\mathrm{K}) / \rho(5 \mathrm{~K})=86$, which is very close to that reported previously, $\mathrm{RRR}=87$ [17]. The upper and lower insets of Fig. 1 show the resistive and magnetic superconducting transitions at low temperature. The middle point of the resistivity transition is at $T_{c}=3.0 \mathrm{~K}$.

In Fig. 2a, the resistivity in $H=0$ and $2 \mathrm{~T}$ are plotted as $\rho$ vs $T^{1.5}$. It is found that $\rho$ obeys $T^{1.5}$ dependence nicely above $T_{c}$, up to about $20 \mathrm{~K}$. Previously, Terashima et al. claimed that $\rho$ exhibits a $T^{2}$ dependence below $\sim$ $45 \mathrm{~K}$ 17. . However, we note that their $T^{2}$ fitting does not look good at low temperature. To elucidate how low the $T^{1.5}$-dependent $\rho(T)$ can go, we measure the resistivity down to $50 \mathrm{mK}$ in a dilution refrigerator and in higher magnetic fields. Fig. $2 \mathrm{~b}$ plots $\rho$ vs $T^{1.5}$ for $H=4,5$, $6,8,11$, and $14.5 \mathrm{~T}$. The downward deviation of $\rho$ from $T^{1.5}$ dependence below $1.6 \mathrm{~K}$ in $H=4 \mathrm{~T}$ is attributed to the onset of superconductivity. In $H=5 \mathrm{~T}$, we find a perfect $T^{1.5}$-dependent resistivity down to $50 \mathrm{mK}$. In $H>5 \mathrm{~T}$, there is an upward deviation of $\rho$ from the $T^{1.5}$ dependence at low temperature. The data of $H=5,6$, 8, 11, and $14.5 \mathrm{~T}$ were re-plotted as $\rho$ vs $T^{2}$ in Fig. 2c. It is clearly seen that a Fermi liquid behavior of resistivity, $\rho \sim T^{2}$, develops with increasing field.

Based on our experimental determined ranges of $T^{2}$ behavior at low temperatures, we have constructed a phase diagram of the $T-H$ plane (Fig. 3). The inset of Fig. 3 plots the field dependence of the coefficient $A$ of the $T^{2}$ term, which tends to diverge towards $H_{c 2}=5 \mathrm{~T}$. Such a phase diagram of $\mathrm{KFe}_{2} \mathrm{As}_{2}$ is strikingly similar to that of the heavy-fermion superconductor $\mathrm{CeCoIn}_{5}$ with $T_{c}=$ $2.3 \mathrm{~K}$, in which a field-induced $\mathrm{AF}$ quantum critical point (QCP) is located at $H_{c 2}$ [18 20].

Near an AF QCP, the scattering of electrons by AFSF usually leads to non-Fermi-liquid behavior of resistivity, $\rho \sim T^{1.5}$ in $3 \mathrm{D}$ system and $\rho \sim T$ in $2 \mathrm{D}$ system 21$]$. The
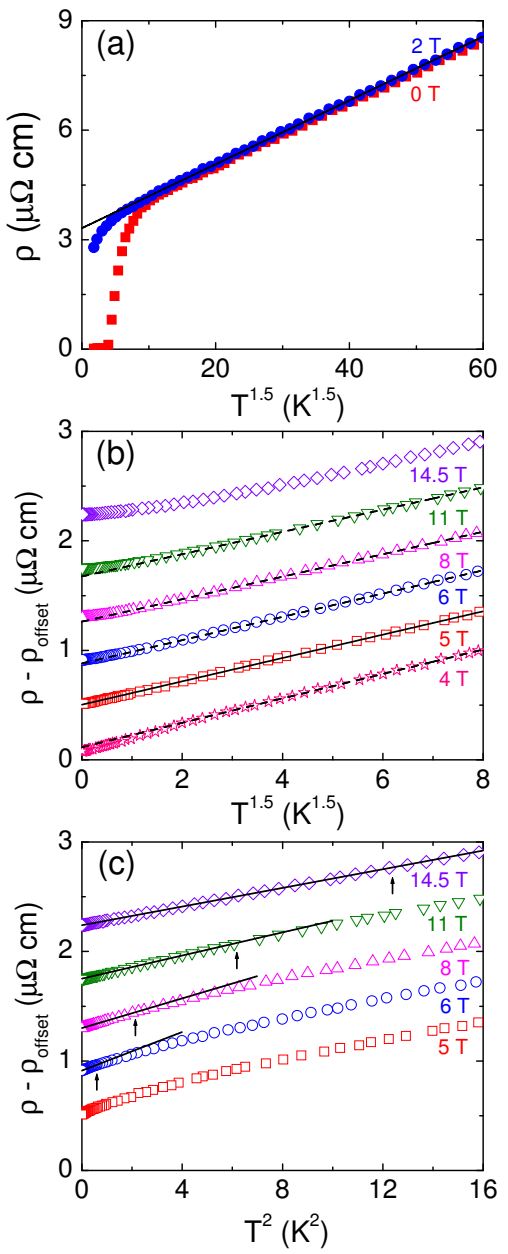

FIG. 2: (Color online). (a) Low-temprature resistivity of $\mathrm{KFe}_{2} \mathrm{As}_{2}$ single crystal in $H=0$ and $2 \mathrm{~T}$ plotted as $\rho$ vs $T^{1.5}$. The solid line is a fit of the $H=2 \mathrm{~T}$ data between 4 and $16 \mathrm{~K}$ to $\rho=\rho_{0}+A T^{1.5}$, which gives residual resistivity $\rho_{0}=3.32 \mu \Omega \mathrm{cm}$. (b) $\rho$ vs $T^{1.5}$ for $H=4,5,6,8,11$, and 14.5 $\mathrm{T}$ (data sets are offset for clarity). The solid line is a fit of the $H=5 \mathrm{~T}$ data between $50 \mathrm{mK}$ and $4 \mathrm{~K}$. The dash lines are guides to the eye for the deviation from the $T^{1.5}$ dependence. (c) $\rho$ vs $T^{2}$ for $H=5,6,8,11$, and $14.5 \mathrm{~T}$ (data sets are offset for clarity). The solid lines are fits to $\rho=\rho_{0}+A T^{2}$. The arrows indicate the upper limit of the temperature range of $T^{2}$ behavior.

observation of $\rho \sim T^{1.5}$ at $H_{c 2}$ in quasi-2D $\mathrm{CeCoIn}_{5}$ is explained by the similar character of the magnetic fluctuations in the $\mathrm{CeIn}_{3}$ planes of $\mathrm{CeCoIn}_{5}$ and in bulk 3D $\mathrm{CeIn}_{3}$ itself [20]. As for $\mathrm{KFe}_{2} \mathrm{As}_{2}$ in this study, the $T^{1.5}$ dependence of $\rho$ at $H_{c 2}$ indicates that the magnetic fluctuations in $\mathrm{KFe}_{2} \mathrm{As}_{2}$ also have some $3 \mathrm{D}$ character, which may need further investigation.

The similarity between the $T-H$ phase diagrams of $\mathrm{KFe}_{2} \mathrm{As}_{2}$ and $\mathrm{CeCoIn}_{5}$ suggests that there is also a fieldinduced AF QCP at $H_{c 2}$ in $\mathrm{KFe}_{2} \mathrm{As}_{2}$. To our knowledge, this is the first time to demonstrate an AF QCP in FeAsbased superconductors down to low temperature.

A field-induced AF QCP at $H_{c 2}$ is very unusual, since 


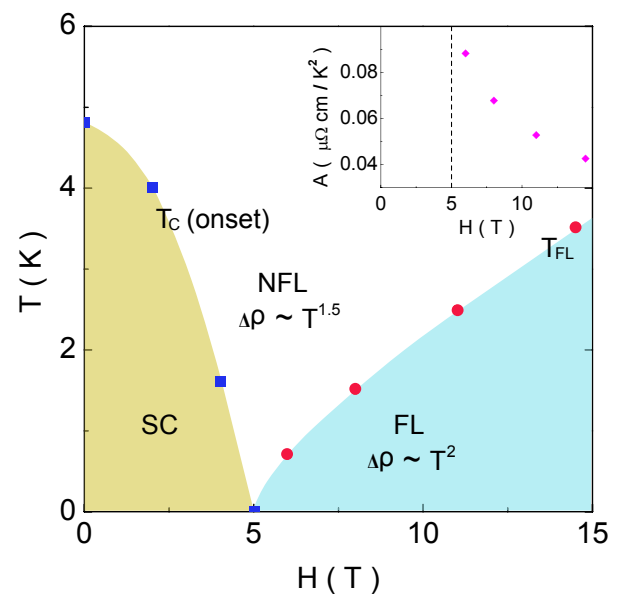

FIG. 3: (Color online). The $T-H$ phase diagram determined from the resistivity measurements. The $T_{c}$ (onset) is defined at the temperature where $\rho$ deviates downwards from the $T^{1.5}$ dependence. The $T_{F L}$ is defined as the upper limit of the temperature range of $T^{2}$ dependence Fermi liquid behavior. This shows a clear field-induced quantum critical point located at $H_{c 2}=5 \mathrm{~T}$. The inset shows the field dependence of the coefficient $A$ of $\rho=\rho_{0}+A T^{2}$, which tends to diverge towards $H_{c 2}=5 \mathrm{~T}$.

it indicates that the superconducting and magnetic orders are tightly coupled [22]. This may be easy to understand in $\mathrm{CeCoIn}_{5}$, since there is a closely related compound $\mathrm{CeRhIn}_{5}$ which is an ambient pressure antiferromagnet with the Néel temperature $T_{N}=3.8 \mathrm{~K}$. For $\mathrm{KFe}_{2} \mathrm{As}_{2}$, this is highly unexpected, in the sense that $\mathrm{KFe}_{2} \mathrm{As}_{2}$ is far away from the $\mathrm{AF}$ parent compound $\mathrm{BaFe}_{2} \mathrm{As}_{2}$ in $\mathrm{Ba}_{1-x} \mathrm{~K}_{x} \mathrm{Fe}_{2} \mathrm{As}_{2}$ system. However, our finding is strongly supported by recent nuclear magnetic resonance (NMR) experiments on $\mathrm{KFe}_{2} \mathrm{As}_{2}$ single crystal, which did claim the existence of strong AFSF [23].

Having demonstrated the field-induced AF QCP in $\mathrm{KFe}_{2} \mathrm{As}_{2}$, we continue to investigate its superconducting gap structure. Fig. 4a shows the temperature dependence of the in-plane thermal conductivity for $\mathrm{KFe}_{2} \mathrm{As}_{2}$ in $H=0,0.1,0.3,0.5,0.8,1.25$, and 2 T magnetic fields, plotted as $\kappa / T$ vs $T$. All the curves are roughly linear, as previously observed in $\mathrm{BaFe}_{1.9} \mathrm{Ni}_{0.1} \mathrm{As}_{2}$ and overdoped $\mathrm{BaFe}_{2-x} \mathrm{Co}_{x} \mathrm{As}_{2}$ single crystals [24 26]. Therefore we fit the data to $\kappa / T=a+b T^{\alpha-1}$ with $\alpha$ fixed to 2 . The two terms $a T$ and $b T^{\alpha}$ represent contributions from electrons and phonons, respectively. Here we only focus on the electronic term.

In zero field, the fitting gives a residual linear term $\kappa_{0} / T=2.27 \pm 0.02 \mathrm{~mW} \mathrm{~K}^{-2} \mathrm{~cm}^{-1}$. Such a large $\kappa_{0} / T$ in $\mathrm{KFe}_{2} \mathrm{As}_{2}$ is really very surprising, since previous thermal conductivity studies of FeAs-based superconductors (without phosphorus), including $\mathrm{Ba}_{0.75} \mathrm{~K}_{0.25} \mathrm{Fe}_{2} \mathrm{As}_{2}$, have given negligible $\kappa_{0} / T$ in $H=0$ 24 27]. From Fig. 4a, a very small field $H=0.1 \mathrm{~T}$ has significantly increased the $\kappa / T$. Above $H=0.8 \mathrm{~T}, \kappa / T$ tends to saturate. In $H=$
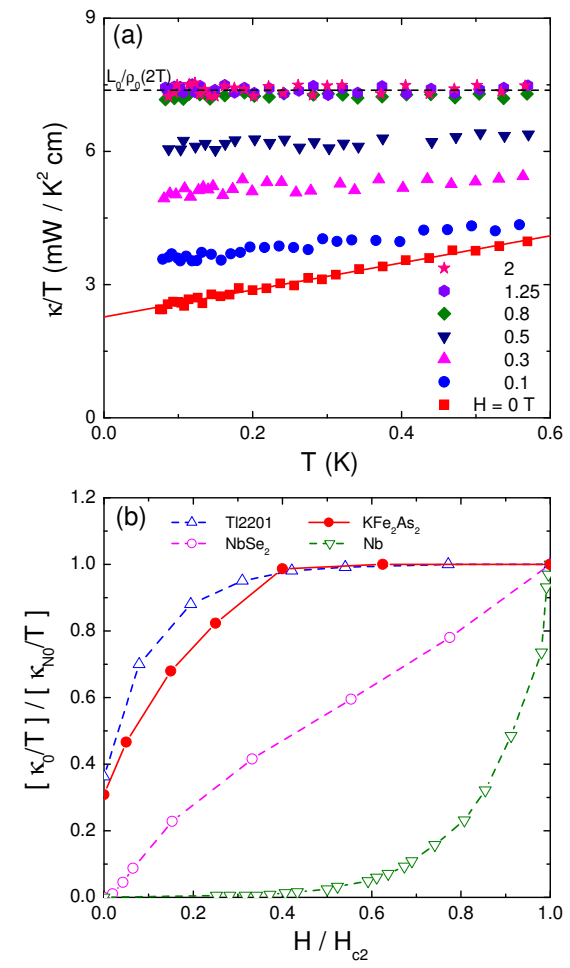

FIG. 4: (Color online). (a) Low-temperature in-plane thermal conductivity of $\mathrm{KFe}_{2} \mathrm{As}_{2}$ in magnetic fields applied along the c-axis $(H=0,0.1,0.3,0.5,0.8,1.25$ and $2 \mathrm{~T})$. The solid line is $\kappa / T=a+b T$ fit to the $H=0 \mathrm{~T}$ data. The dash line is the normal state Wiedemann-Franz law expectation $L_{0} / \rho_{0}(2$ $\mathrm{T})$, with $L_{0}$ the Lorenz number $2.45 \times 10^{-8}{\mathrm{~W} \Omega \mathrm{K}^{-2}}^{2}$ and $\rho_{0}(2 \mathrm{~T})=3.32 \mu \Omega \mathrm{cm}$. (b) Normalized residual linear term $\kappa_{0} / T$ of $\mathrm{KFe}_{2} \mathrm{As}_{2}$ as a function of $H / H_{c 2}$. Similar data of the clean $s$-wave superconductor $\mathrm{Nb}$ [28], the multi-band $s$ wave superconductor $\mathrm{NbSe}_{2}$ [29], and an overdoped sample of the $d$-wave superconductor Tl-2201 [30] are also shown for comparison. The behavior of $\kappa_{0}(H) / T$ in $\mathrm{KFe}_{2} \mathrm{As}_{2}$ clearly mimics that in Tl-2201.

1.25 and $2 \mathrm{~T}, \kappa_{0} / T=7.39 \pm 0.03$ and $7.36 \pm 0.04 \mathrm{~mW}$ $\mathrm{K}^{-2} \mathrm{~cm}^{-1}$ were obtained from the fittings, respectively. Both values meet the expected normal state WiedemannFranz law expectation $L_{0} / \rho_{0}(2 \mathrm{~T})=7.38 \mathrm{~mW} \mathrm{~K}^{-2} \mathrm{~cm}^{-1}$, within experimental error bar. We take $H=2 \mathrm{~T}$ as the bulk $H_{c 2}$ of $\mathrm{KFe}_{2} \mathrm{As}_{2}$, despite that the resistive transition is not completely suppressed until $H_{c 2}$ (onset) $=5 \mathrm{~T}$. To choose a slightly different bulk $H_{c 2}$ does not affect our discussions below.

In Fig. 4b, the normalized $\kappa_{0} / T$ of $\mathrm{KFe}_{2} \mathrm{As}_{2}$ is plotted as a function of $H / H_{c 2}$, together with the clean $s$-wave superconductor $\mathrm{Nb}$ [28], the multi-band $s$-wave superconductor $\mathrm{NbSe}_{2}$ [29], and an overdoped sample of the $d$-wave superconductor Tl-2201 [30]. For $\mathrm{KFe}_{2} \mathrm{As}_{2}$, the large $\kappa_{0} / T$ in $H=0$ and the rapid increase of $\kappa_{0} / T$ at low field mimic the behavior of Tl-2201, and provide clear evidences for unconventional superconducting gap with nodes [15]. We note that recent ${ }^{75}$ As nuclear quadrupole resonance (NQR) and specific heat measure- 
ments on $\mathrm{KFe}_{2} \mathrm{As}_{2}$ polycrystals also suggested multiple nodal gaps [31].

The nodal gap in extremely hole-doped $\mathrm{KFe}_{2} \mathrm{As}_{2}$ is distinctly different from the nodeless gaps in FeAs-based superconductors at other doping [24 27, 32, 33]. In optimally hole-doped $\mathrm{Ba}_{0.6} \mathrm{~K}_{0.4} \mathrm{Fe}_{2} \mathrm{As}_{2}$ and electron-doped $\mathrm{BaFe}_{1.85} \mathrm{Co}_{0.15} \mathrm{As}_{2}$, the observations of nearly nested Fermi-surface pockets and nodeless gaps suggest that the interband interaction may play a crucial role in superconducting pairing [32,33], thus support the $s_{ \pm}$-wave pairing mechanism in FeAs-based superconductors [7]. However, in $\mathrm{KFe}_{2} \mathrm{As}_{2}$, the electron pockets at $\mathrm{M}$ point completely disappear due to hole doping [14]. Although four new small hole $(\epsilon)$ pockets are found around M point, the interband interaction is nevertheless suppressed, therefore the $s_{ \pm}$-wave pairing mechanism does not work in $\mathrm{KFe}_{2} \mathrm{As}_{2}$ [14]. Having known that there exist strong AFSF near the AF QCP, one can be sure that the nodal superconductivity in $\mathrm{KFe}_{2} \mathrm{As}_{2}$ results from intraband pairing via AFSF. Usually, the pairing mediated by AFSF has $d$-wave symmetry, as in $\mathrm{CeCoIn}_{5}$ [34]. Because of the great similarity between the $T-H$ phase diagrams of $\mathrm{KFe}_{2} \mathrm{As}_{2}$ and $\mathrm{CeCoIn}_{5}$, and the similar behavior of $\kappa_{0}(H) / T$ between $\mathrm{KFe}_{2} \mathrm{As}_{2}$ and $\mathrm{Tl}-2201$, the nodal gap in $\mathrm{KFe}_{2} \mathrm{As}_{2}$ is very likely also $d$-wave.

We note that there is an electron-hole asymmetry in the phase diagram of FeAs-based superconductors at the heavily overdoped regime. For the heavily electron-doped $\mathrm{BaFe}_{1.7} \mathrm{Co}_{0.3} \mathrm{As}_{2}$, the hole pocket at $\Gamma$ point completely disappears [35], and the interband interaction is also suppressed. But it turns out that $\mathrm{BaFe}_{1.7} \mathrm{Co}_{0.3} \mathrm{As}_{2}$ is not superconducting [35]. This electron-hole asymmetry may be explained by the different strength of AFSF on electron- and hole-doped sides measured by NMR [23, 36]. In $\mathrm{BaFe}_{2-x} \mathrm{Co}_{x} \mathrm{As}_{2}$, the spin fluctuations are completely suppressed at $\mathrm{x}>0.3$ [36], while strong AF spin fluctuations were found in $\mathrm{KFe}_{2} \mathrm{As}_{2}$ [23].

Finally, it is worth to point out that the possible nodal superconductivity in $\mathrm{LaFePO}$ and $\mathrm{BaFe}_{2}\left(\mathrm{As}_{1-x} \mathrm{P}_{x}\right)_{2}$ 13. may be different from that in $\mathrm{KFe}_{2} \mathrm{As}_{2}$. The main reason is that the isovalent substitution of $\mathrm{P}$ for As only slightly modifies the Fermi surface [12], therefore the Fermi-surface nesting and interband interaction still exist in these two compounds. To get nodal superconductivity in $\mathrm{LaFePO}$ and $\mathrm{BaFe}_{2}\left(\mathrm{As}_{1-x} \mathrm{P}_{x}\right)_{2}$, one needs to consider the competition between $s_{ \pm}$-wave and $d$-wave superconducting states even more carefully.

In summary, we have measured the resistivity and thermal conductivity of extremely hole-doped $\mathrm{KFe}_{2} \mathrm{As}_{2}$ single crystal down to $50 \mathrm{mK}$. A field-induced AF QCP is demonstrated by the observation of $\rho \sim T^{1.5}$ at $H_{c 2}=5$ $\mathrm{T}$, and the development of $\rho \sim T^{2}$ Fermi liquid behavior at $H>5 \mathrm{~T}$. Furthermore, the large $\kappa_{0} / T$ at zero field and a rapid increase of $\kappa_{0}(H) / T$ at low field give strong evidences for nodal superconductivity in $\mathrm{KFe}_{2} \mathrm{As}_{2}$. Such a nodal superconductivity, very likely $d$-wave, naturally results from the intraband pairing via AFSF near the AF QCP. Our results are consistent with the suppression of interband interaction in $\mathrm{KFe}_{2} \mathrm{As}_{2}$, as revealed by ARPES experiments.

We thank Y. Chen, D. L. Feng, and Y. Y. Wang for discussions. This work is supported by the Natural Science Foundation of China, the Ministry of Science and Technology of China (National Basic Research Program No:2009CB929203), Program for New Century Excellent Talents in University, and STCSM of China (No:08dj1400200 and 08PJ1402100).

* E-mail: shiyan_li@fudan.edu.cn

[1] Y. Kamihara et al., J. Am. Chem. Soc. 130, 3296 (2008).

[2] X. H. Chen et al., Nature 453, 761-762 (2008).

[3] M. Rotter, M. Tegel and D. Johrendt, Phys. Rev. Lett. 101, 107006 (2008).

[4] R. H. Liu et al., Phys. Rev. Lett. 101, 087001 (2008).

[5] C. de la Cruz et al.,Nature 453, 899-902 (2008).

[6] Q. Huang et al.,Phys. Rev. Lett. 101, 257003 (2008).

[7] I. I. Mazin et al., Phys. Rev. Lett. 101, 057003 (2008).

[8] K. Kuroki et al., Phys. Rev. Lett. 101, 087004 (2008).

[9] I. I. Mazin and J. Schmalian, Physica C 469, 614 (2009).

[10] J. D. Fletcher et al., Phys. Rev. Lett. 102, 147001 (2009).

[11] C. W. Hicks et al., Phys. Rev. Lett. 103, 127003 (2009).

[12] K. Hashimoto et al., arXiv:0907.4399.

[13] Y. Nakai et al., Phys. Rev. B 81, 020503(R) (2010).

[14] T. Sato et al., Phys. Rev. Lett. 103, 047002 (2009).

[15] H. Shakeripour et al., New J. Phys. 11, 055065 (2009).

[16] X. F. Wang et al., Phys. Rev. Lett. 102, 117005 (2009).

[17] T. Terashima et al., J. Phys. Soc. Jpn. 78, 063702 (2009).

[18] J. Paglione et al., Phys. Rev. Lett. 91, 246405 (2003).

[19] A. Bianchi et al., Phys. Rev. Lett. 91, 257001 (2003).

[20] J. Paglione et al., Phys. Rev. Lett. 97, 106606 (2006).

[21] G. R. Stewart, Rev. Mod. Phys. 73, 797 (2001).

[22] M. Kenzelmann et al., Science 321, 1652-1654 (2008).

[23] S. W. Zhang et al., Phys. Rev. B 81, 012503 (2010).

[24] L. Ding et al., New J. Phys. 11, 093018 (2009).

[25] M. A. Tanatar et al., arXiv:0907.1276

[26] J. K. Dong et al., arXiv:0908.2209.

[27] X. G. Luo et al., Phys. Rev. B 80, 140503(R) (2009).

[28] J. Lowell and J. Sousa, J. Low. Temp. Phys. 3, 65 (1970).

[29] E. Boaknin et al., Phys. Rev. Lett. 90, 117003 (2003).

[30] C. Proust et al., Phys. Rev. Lett. 89, 147003 (2002).

[31] H. Fukazawa et al., J. Phys. Soc. Jpn. 78, 083712 (2009).

[32] P. Richard et al., Phys. Rev. Lett. 102, 047003 (2009).

[33] K. Terashima et al., Proc. Natl. Acad. Sci. 106, 7330 (2009).

[34] A. Vorontsov and I. Vekhter, Phys. Rev. Lett. 96, 237001 (2006).

[35] Y. Sekiba et al., New J. Phys. 11, 025020 (2009).

[36] F. L. Ning et al., Phys. Rev. Lett. 104, 037001 (2010). 